\title{
Survey on a Novel Wireless Sensor Network Communication at 433MHz Frequency
}

\author{
Xiaoqing Yu, Wengting Han* and Zenglin Zhang \\ ${ }^{1}$ Northwest A \& F University, Shaanxi, Yangling, 712100, China \\ ${ }^{2}$ Research Institute of Water-saving Agriculture of Arid Regions of China, \\ Shaanxi, Yangling, 712100, China \\ E-mail: yuxiaoqing115@gmail.com \\ wentinghan200@126.com;zhangzenglin115@gmail.com
}

\begin{abstract}
Wireless underground sensor networks (WUSN) are a promising new technology for monitoring soil information with a high spatial and temporal resolution for large areas. However, the significantly high attenuation caused by soil is the main challenge for the feasibility of WUSN. Recent theoretical results highlight the potential of smaller attenuation rates with the use of smaller radio frequencies. In this paper, experimental measurements have been conducted with commodity sensor nodes at the frequency of 433 $\mathrm{MHz}$. Experiments are run to examine the received signal strength of correctly received packets and the packet error rate for a communication link. The tests show the potential feasibility of the WUSN with the use of powerful RF transceivers at $433 \mathrm{MHz}$ frequency. Moreover, we also illustrate a classification for wireless underground sensor network communication. Finally, we conclude that the effects of burial depth, inter-node distance and volumetric water content of the soil on the signal strength and packet error rate.
\end{abstract}

Keywords: Wireless sensor networks, Experiment, Communication, Transmission, Error rate

\section{Introduction}

Wireless underground sensor networks are a specialized kind of Wireless Sensor Networks (WSN) that mainly focuses on the use of sensors that communicate through soil and are a natural extension of the WSN phenomenon to the underground environment $[1$, 2]. Wireless underground sensor network is that the WUSN sensor equipment with wireless transceiver module buried completely in certain soil depth, sensor module percept the data and send data through the wireless mode. Many sensor nodes consist of sensor network in the soil and complete automatically the entire process of data perception, collection. WUSN have several remarkable merits, such as concealment, ease of deployment, timeliness of data, reliability and coverage density. Besides monitoring soil ingredients in underground, wireless underground sensor network can also be used for monitoring soil motion, forecasting landslide, underground ice motion and volcanic eruptions, and it has higher value for study [3-6]. Despite their potential advantages, however, the realization of WUSN is challenge due to the significant and direct impact of soil characteristics and its dynamics on communication. This is possible by exploiting real-time soil condition information from a network of underground sensors.

Given the usefulness of monitoring conditions in the underground [7-9], we set out to determine whether current wireless sensor networks solutions are applicable to the underground sensing environment. In this paper, the results of experiments for wireless underground sensor networks at the frequency of $433 \mathrm{MHz}$ using commodity sensor nodes is presented. Moreover, lessons learned from these experiments for the received signal strength and the packet error rate of efficient communication for WUSN are discussed. 
The results of the experiments show the potential feasibility of the WUSN with the use of commodity motes.

The rest of this paper is organized as follows: In Section 2, the related work of the wireless underground sensor networks technology is given. In Section 3, the materials for the experiments and the experimental methodology are described in Section3. The experiment results for the collection of irrigation information using WUSN motes are presented in Section 4. Finally, the summary of the paper and its key contributions are discussed in Section 5.

\section{Related Work}

Wireless underground sensor networks are a new research subject, at present, it is in the experimental study phase and also no mature products are in the market. Research reports of wireless underground sensor networks in agricultural application are little, the present study include mainly path loss, bit error rate, maximum transmission distance, test error of water content of path transmission of the electromagnetic wave under the main influence factors, these factors are soil types, volumetric water content of the soil, depth of nodes buried, internodes distance, the range of frequency, etc., [10-16].

Network system structure of wireless underground sensor networks system aiming at intelligent transportation system and maintenance of the near surface soil (such as golf courses, a football field) was designed in [17]. The software and hardware systems of the nodes were also designed. In addition, the collect nodes used the low performance microcontroller; the receiving nodes on the ground used the high performance microcontroller, development and testing research of network system were not carried; In the [17], there is also studied that the performance of the wireless underground sensor networks which was influenced by propagation of electromagnetic waves in the soil, underground channel model, electrical characteristics of soil and deployed solutions of wireless underground sensor networks nodes. In $400 \mathrm{MHz}$ frequency, sensor buried depth $0.5 \mathrm{~m}$, horizontal spacing of sensor $1 \mathrm{~m}$, conductivity 0.1 and dielectric constant 10 under, transmission parameters of electromagnetic wave and energy losses for different volumetric water content of the soil, different proportion sand and clay soil were analyzed through MATLAB mathematical simulation software.

In the laboratory of [18], signal attenuation of ZigBee wireless transceiver module of the $2.44 \mathrm{GHz}$ frequency was researched by using soil column in different soil types and the water content. Experimental results showed that increase of soil column depth and volumetric water content of the soil could lead to increase of signal attenuation, the relationship could be expressed in linear model, and the correlation coefficient is greater than 0.9 .

Wireless underground sensor networks have been investigated in many contexts recently. The concept of WUSN and the challenges related to the underground wireless channel have been introduced in [3,4]. In the wireless underground sensor network communication, the sensor nodes are deployed mainly in the underground soil, but it still need to communicate with the ground node to implement the data collection, management and relay, etc. Therefore, there are three different kinds of WUSN communication mode based on the transmitting node and the receiving node are deployed on the aboveground or underground in the soil, the aboveground-underground communication, undergroundaboveground and underground-underground communication [19-22]. Accordingly, three different communication links exist in WUSN based no the locations of the transmitter and receiver as shown in Figure 1. 


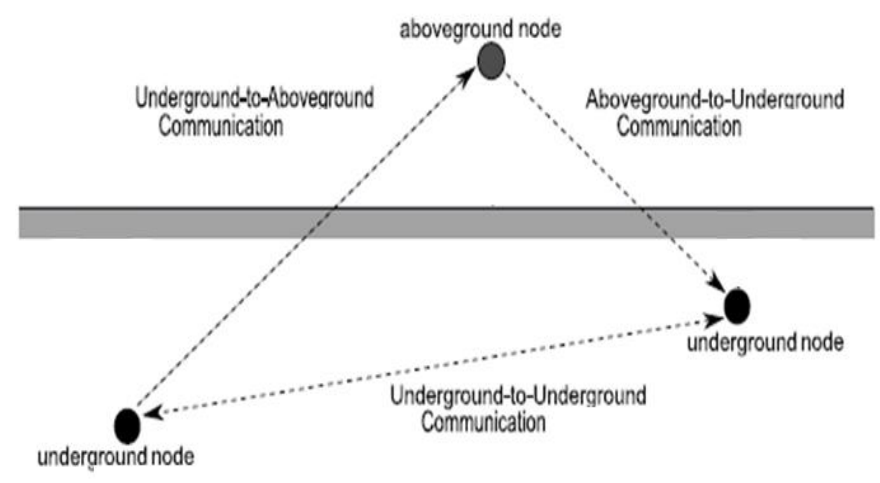

Figure 1. Types of Communication in WUSN

As shown in Figure 1, communication types of the WUSN can be mainly classified into three: aboveground-to-underground link: aboveground sender node sends messages to underground nodes, underground-to-aboveground link: the sender is buried and the receiver is above the ground and underground-to-underground link: both the sender and the receiver are buried underground and communicate through soil. Underground-toaboveground link and aboveground-to-underground link are required for several functionalities of WUSN, such as network management and data retrieval. Thus, the characterization of the bi-directional communication between a buried node and an aboveground device is essential. Both the underground-to-aboveground link and aboveground-to-underground link include underground propagation. Thus, undergroundto-underground link is also very important. Moreover, the soil properties, such as soil moisture, directly impact the communication success [23, 24]. Furthermore, the soil-air interface plays an important role in communication. Transmitted rays are reflected and attenuated at this interface, which significantly influences the communication quality.

Furthermore, we consider agricultural applications of WUSN, which usually require burial depths greater due to plowing and similar mechanical activities occur at the soil $[25,26]$. Accordingly, the majority of the experiments consider a better burial depth. In this work, we provide the effects of the node burial depth, horizontal inter-node distance and soil water content on the communication of aboveground to underground, underground to aboveground and underground to underground.

\section{Materials and Methodology}

The signal propagation in soil depends on the path loss in soil. Received power as a function of transmitted signal, path loss and antenna gain at the receiver end is given from Friis equation as shown in Equation (1) [27].

$$
P_{r}(d B m)=P_{t}(d B m)+G_{t}(d B)+G_{r}(d B)-L_{p h}(d B)
$$

where $P_{t}$ is the transmitter power, $G_{t}$ and $G_{r}$ are the gains of the transmitter and receiver antenna, $\mathrm{L}_{\mathrm{ph}}$ is the path loss in soil. The path loss is shown in Equation (2) [28].

$$
L_{p h}(d B)=L_{0}(d B)+L_{c}(d B)+L_{a}(d B)
$$

$\mathrm{L}_{0}$ is the path loss in air and given in Equation (3).

$$
L_{0}(d B)=20 \log \left(\frac{4 \pi d f}{c}\right)
$$

where $\mathrm{d}$ is the distance between transmitter and receiver in meter, $\mathrm{f}$ is the operating frequency in Hertz and $\mathrm{c}$ is the velocity of light in air in meter per second.

$L_{c}(d B)$ is the path loss due to changing in medium and given by Equation (4) [29]. 


$$
L_{c}(d B)=20 \log \left(\frac{\lambda_{0}}{\lambda}\right)
$$

where $\lambda_{0}$ is the signal wavelength in air and calculated $\left(\lambda_{0}=\mathrm{c} / \mathrm{f}\right)$ and $\lambda$ is the wave factor and given by $(\lambda=2 \pi / \beta)$ and $\beta$ is the phase shifting constant and calculated as shown in Equation (5).

$$
\beta=\omega \sqrt{\frac{\mu \varepsilon^{\prime}\lceil}{2}\left\lfloor\sqrt{1+\left(\frac{\varepsilon^{\prime \prime}}{\varepsilon^{\prime}}\right)^{2}}+1\right\rfloor}
$$

where $\varepsilon^{\prime}$ and $\varepsilon^{\prime \prime}$ are the real and imaginary parts of the complex dielectric constant given by $\left(\varepsilon=\varepsilon^{\prime}-j \varepsilon^{\prime \prime}\right)$.

$\mathrm{L}_{\mathrm{a}}(\mathrm{dB})$ is the path loss due to attenuation in medium and given by Equation (6).

$$
L_{a}(d B)=10 \log \left(e^{-2 \alpha d}\right)
$$

where $\alpha$ is the attenuatin constant and calculated as shown in Equation (7).

$$
\alpha=\omega \sqrt{\frac{\mu \varepsilon^{\prime}}{2}\left\lfloor\sqrt{1+\left(\frac{\varepsilon^{\prime \prime}}{\varepsilon^{\prime}}\right)^{2}}-1 !\right.}
$$

In the trial, we assume the clay percent as $15 \%$,the silt percent as $35 \%$, the sand particle percent as $50 \%$, the bulk density as $1.5 \mathrm{~g} / \mathrm{cm}^{3}$. In this section, the details of the outdoor environment hardware, software, and the methodology for the experiments are presented. The underground experiments with $433 \mathrm{MHz}$ sensor nodes were carried out in the laboratory. To observe the effects of soil moisture, two different volumetric water content values are considered. Experiments realized in dry and wet conditions correspond to volumetric water content of $10 \%$ and $30 \%$, respectively. For the experiments, nodes operate at $433 \mathrm{MHz}$ band are used. The node radio supports variable output power, the radio was always set to its maximum transmit power of $10 \mathrm{dBm}$. The size of each packet is 37 bytes and a $100 \mathrm{~ms}$ delay between each packet transmission is configured. The antenna of node is a standard one-quarter wavelength monopole antenna with $17 \mathrm{~cm}$ lengths, and the antennas are vertically oriented.

The experiments were designed to collect packet error rates at the application layer, as well as the received signal strength indicator of correctly received packets. Each experiment in this work is based on a set of 3 tests with 350 messages or 2 experiments with 500 messages, which result in a total of 1000 packets. The number of packets correctly received by one or more receiver nodes is recorded along with the signal strength for each packet. Accordingly, the packet error rate and the received signal strength level from each receiver are collected. To prevent the effects of hardware failures of each individual node, qualification tests have been performed before each experiment.

\section{Experiment Results}

Experiments are conducted in the above condition. It can been concluded that node burial depth, the inter-node distance and the soil volumetric water content have important effect on the WUSN communication. The results are presented considering how some parameters affect the wireless underground sensor networks communication: the burial depth, the inter-node distance and volumetric water content of the soil. 


\subsection{Tests of Burial Depth}

To investigate the effects of burial depth on the signal strength and packet error rate, the horizontal distance between the sender and receiver is fixed $40 \mathrm{~cm}$. In the communication between the aboveground node and underground node, the aboveground sensor node was on the surface of the ground, the depth of the underground node is varied from $10 \mathrm{~cm}$ to $100 \mathrm{~cm}$. While one node fixed depth $40 \mathrm{~cm}$, another node is varied from 10 $\mathrm{cm}$ to $100 \mathrm{~cm}$ in the underground-to-underground communication. In the Figure 2, the received signal strength and error rate values are shown, respectively, as a function of the receiver depth.

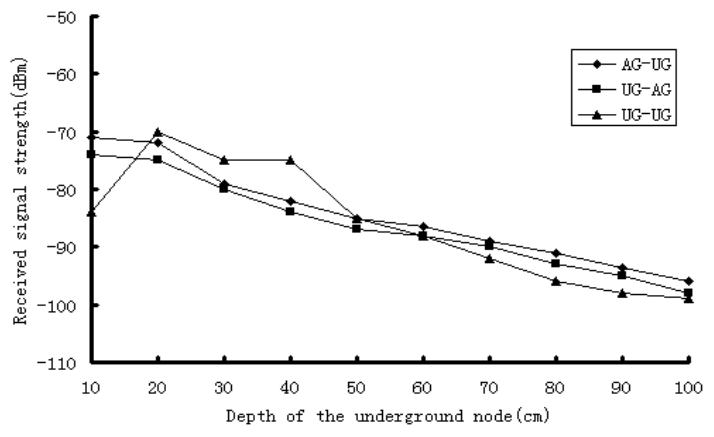

(a) Received Signal Strength vs. Depth

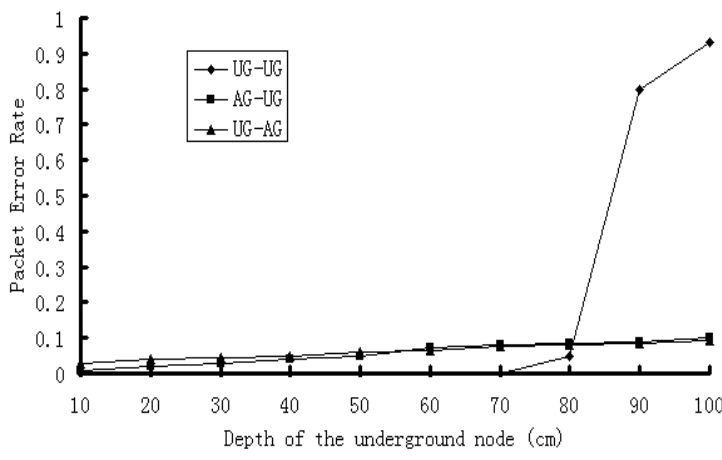

(b) Packet Error Rate vs. Depth

Figure 2. Tests for the Received Signal Strength and Packet Error Rates. The Depth of Underground Node is Varied from $10 \mathrm{~cm}$ to $100 \mathrm{~cm}$

It can be seen from Figure 2 that node burial depth plays an important role in the aboveground-to-underground communication, underground-to-aboveground communication and underground-to-underground communication. The experiments show that a higher burial depth also implies an increase in the soil path, higher attenuation is observed at higher depth. It well known that EM waves that propagate from a medium with a lower dielectric constant such as air to other one with a higher constant such as soil are highly reflected. Consequently, the air-soil interface causes an additional attenuation for the communication. Moreover, the shallower burial depth can significantly enhance aboveground-to-underground communication. When the node burial depth changes from $10 \mathrm{~cm}$ to $60 \mathrm{~cm}$, the packet error rate of aboveground-to-underground communication is lower than underground-to-aboveground communication. While the packet error rate of underground-to-aboveground communication increases begin in the depth $60 \mathrm{~cm}$. Furthermore, it can be observed in the Figure 2 (b), that for the underground node burial depth $80 \mathrm{~cm}$, the packet error rate increases and an increase in burial depth to $100 \mathrm{~cm}$ results in a communication loss. Note that the burial depth plays an important role in the connectivity of the WUSN design. 


\subsection{Tests of Inter-node Distance}

To illustrate the effects of the inter-node distance on the signal strength and packet error rate, experiments are performed with the fixed burial depth $40 \mathrm{~cm}$ of the WUSN node, and the inter-node distance is varied from $10 \mathrm{~cm}$ to $100 \mathrm{~cm}$. In the communication between the aboveground node and underground node, the aboveground sensor node was on the surface of the ground, the depth of the underground node was fixed $40 \mathrm{~cm}$, whereas both of nodes are fixed depth $40 \mathrm{~cm}$ in the underground-to-underground communication. In the tests, the horizontal inter-node distance is varied from $10 \mathrm{~cm}$ to $100 \mathrm{~cm}$. As shown in Figure 3 (a) and (b), the received signal strength and packet error rate values are shown, respectively, as a function of the horizontal inter-node distance.

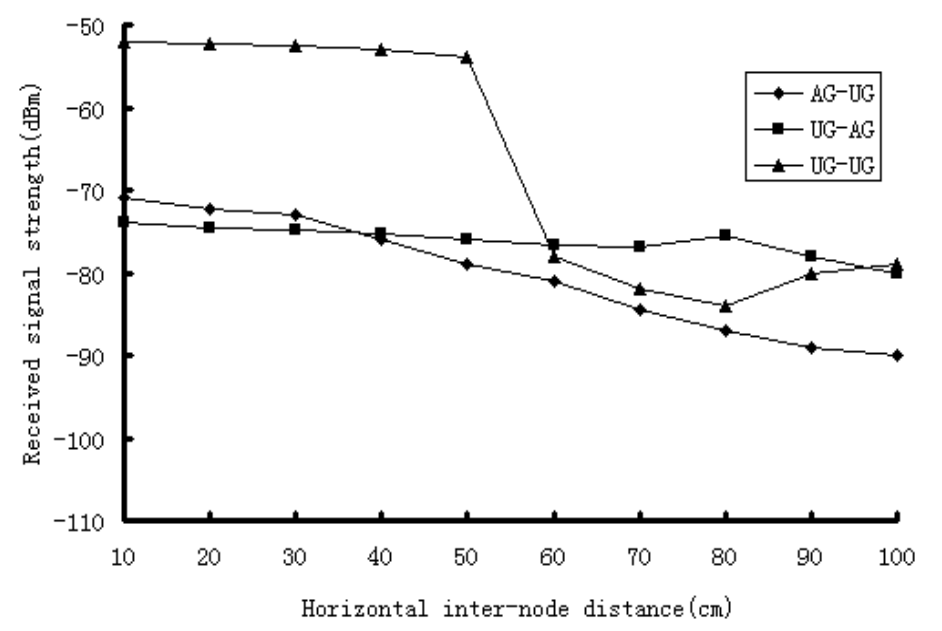

(a) Received Signal Strength

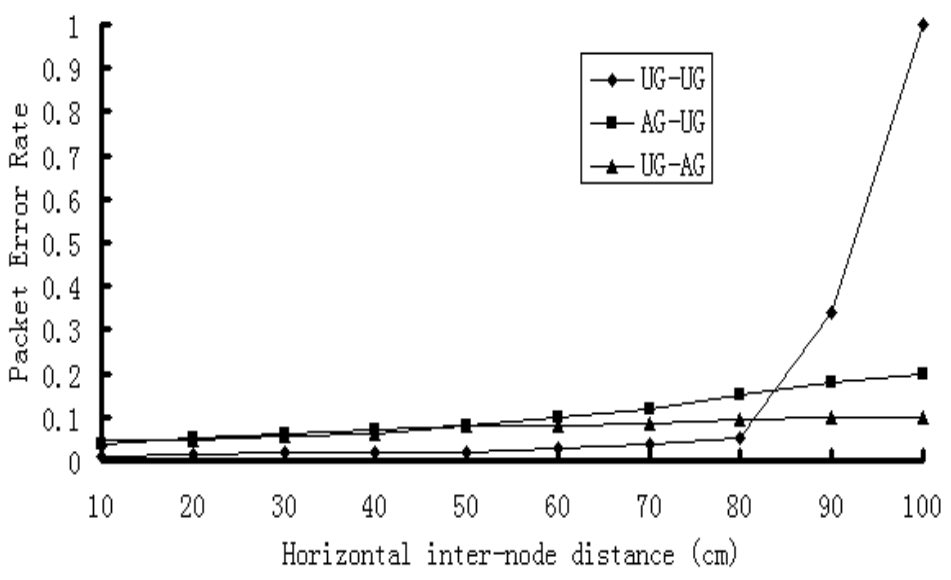

(b) Packet Error Rate

\section{Figure 3. Test for the Received Signal Strength and Packet Error Rates. The Inter-node Distance is Varied from $10 \mathrm{~cm}$ to $100 \mathrm{~cm}$}

In the communication between aboveground and underground, the received signal strength of aboveground-to-underground communication is higher than underground-toaboveground communication when horizontal inter-node distance is smaller than $40 \mathrm{~cm}$. When the horizontal inter-node distance is change from $40 \mathrm{~cm}$ to $100 \mathrm{~cm}$, undergroundto-aboveground communication is higher. At the same time, the packet error rate of aboveground and underground communications are nearly the same when the horizontal 
inter-node distance is smaller than $60 \mathrm{~cm}$. From the whole, both of the packet error rates are less than $20 \%$.

For the underground-to-underground communication, when the inter-node distance varies from $50 \mathrm{~cm}$ to $60 \mathrm{~cm}$, the significant decrease of the signal strength can be observed in Figure 3 (a). The maximum horizontal inter-node distance is found to be between $80 \mathrm{~cm}$ and $90 \mathrm{~cm}$ as shown in Figure 3 (b).

\subsection{Tests of Volumetric Water Content of the Soil}

In the section, the tests of the volumetric water content on the received signal strength and packet error rate are discussed. In the communication between the aboveground node and underground node, the aboveground sensor node was on the surface of the ground, the depth of the underground node was fixed $40 \mathrm{~cm}$, whereas both of nodes are fixed depth $40 \mathrm{~cm}$ in the underground-to-underground communication. In the experiments, the horizontal inter-node distance changes from $10 \mathrm{~cm}$ to $100 \mathrm{~cm}$ with two different volumetric water content levels $10 \%$ and 30\%, respectively. As shown in Figure 4 and Figure 5, the received signal strength and packet error rate values are shown, respectively, as a function of the horizontal inter-node distance with different volumetric water content levels.

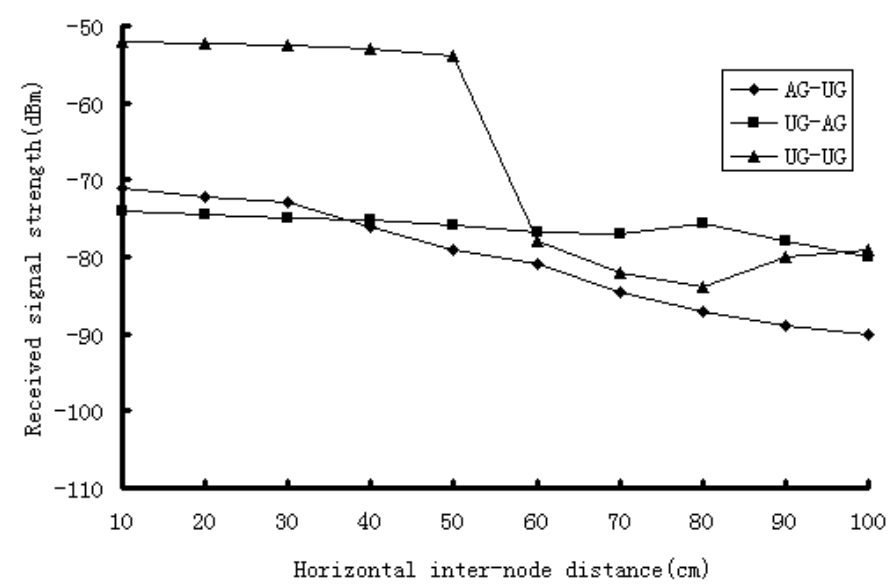

(a) Received Signal Strength

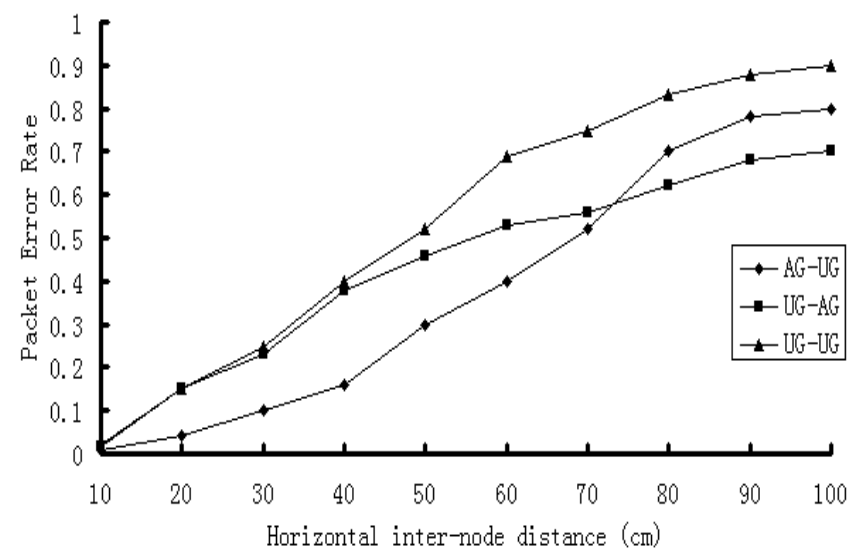

(b) Packet Error Rate

Figure 4. Test for the Received Signal Strength and Packet Error Rates. The Volumetric Water Content Levels 10\% 


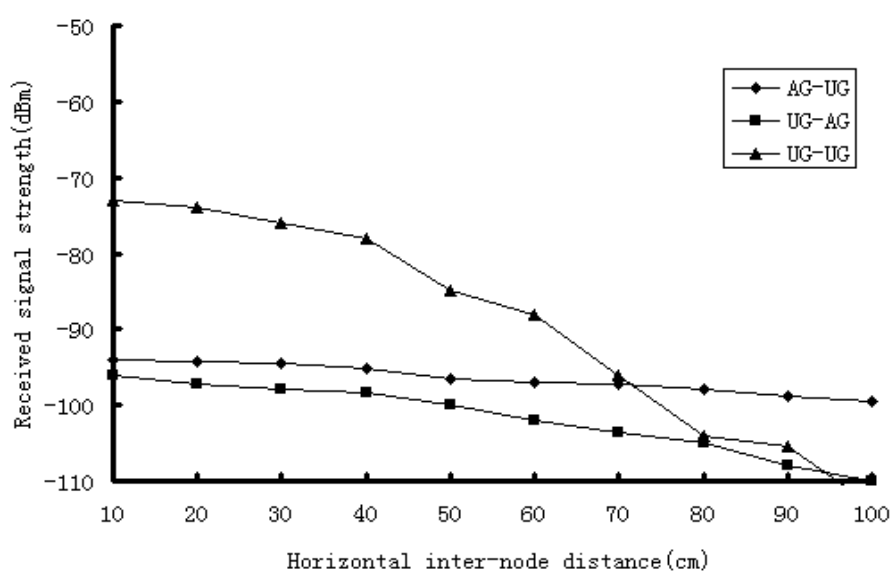

(a) Received Signal Strength

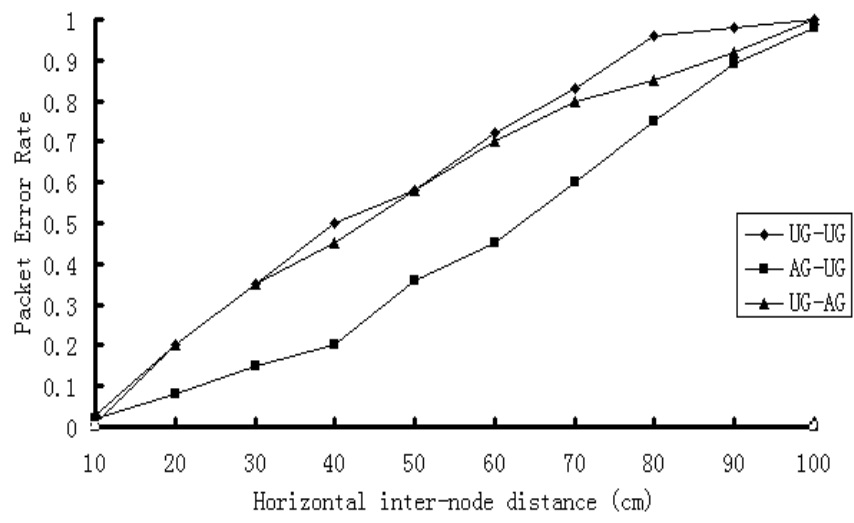

(b) Packet Error Rate

\section{Figure 5. Test for the Received Signal Strength and Packet Error Rates. The} Volumetric Water Content Levels $30 \%$

As shown in Figure 4 (a) and Figure 5 (a), the variation from $10 \%$ to $30 \%$ in the volumetric water content level causes an additional attenuation of $7 \mathrm{~dB}$, on the average, for both underground-to-aboveground and aboveground-to-underground communication. For underground-to-underground communication, the received signal strength decreases about $10 \mathrm{~dB}$. We can also observe, from the Figure 4 (b) and Figure 5 (b), that the negative effect of the volumetric water content over the quality of the communication is reduced when the horizontal inter-node distance is increased. An increase in the soil moisture has two main effects on the wireless underground sensor networks communication. First, the wavelength of the signal is decreased, which causes indirect loss due to the antenna mismatch. Second, an increase in soil moisture significantly increases the soil attenuation. This result shows that soil moisture has a significant influence on the quality of WUSN communication. Therefore, the design of the WUSN protocols should carefully adapt to the variation of the volumetric water content of the soil.

\section{Conclusions}

Despite their potential, the proliferation of WUSN has been delayed by the unique challenges of the underground environment. Some of these challenges are related to the communication involving an underground node and an aboveground device. In this paper, we propose a classification of wireless underground sensor networks communication and present experiment results for aboveground-to-underground, underground-toaboveground and underground-to-underground communication for WUSN. The work 
provides insight to communication through soil using commodity sensor nodes. The experiment results reveal the feasibility of using commodity terrestrial sensor motes for WUSN. Moreover, the experiment results show that the burial depth is important for the WUSN tests due to the effects of reflected rays from the underground-air interface at the surface. In addition, we have shown that the inter-node distance plays an important role in the communication of WUSN. Finally, the direct influence of volumetric water content of the soil on the communication success is shown. These results have a significant impact on the development of multi-hop networking protocols for WUSN.

In addition to the characteristics of wireless underground sensor networks communication, the limitations of the commodity sensor nodes for WUSN are also observed as a result of these experiments. It can be observed that for this specific burial depth, the inter-node distance was smaller than $1 \mathrm{~m}$. Consequently, we expect that a new generation of nodes with more powerful transceivers and more efficient antennas are required for the actual deployment of WUSN applications.

\section{Acknowledgements}

The authors wish to thank the National Engineering Research Center for Water-Saving Irrigation, which partially supported this research through the "China Postdoctoral Science Foundation funded project (2014M552495)" and the Program for New Century Excellent Talents in University (NECT-12-0473) from the Ministry of Education. The authors are also grateful to the anonymous reviewers for their valuable feedback.

\section{References}

[1] A. R. Silva and M. C. Vuran, "Communication with above devices in wireless underground sensor networks: an empirical stud", IEEE International Conference Proceedings, (2010), pp. 23-27.

[2] L. Li, M. C. Vuran and I. F. Akyildizy, "Characteristics of Underground Channel for Wireless Underground Sensor Networks", The Sixth Annual Mediterranean Ad Hoc Networking Workshop, vol. 6, (2007), pp. 12-15.

[3] I. F. Akyildiz, M. C. Vuran and Z. Sun, "Channel modeling for Wireless Underground Communication in Soil", Physical Communication, (2009).

[4] I. F. Akyildiz and E. P. Stuntebeck, "Wireless underground sensor networks: Research challenges", Ad Hoc Networks, vol. 4, (2006), pp. 669-686.

[5] Z. Sun and I. F. Akyildiz, "Channel Modeling of Wireless Networks in Tunnels", IEEE Globecom, New Orleans, USA, (2008).

[6] I. F. Akyildiz, W. Su, Y. Sankarasubramaniam and E. Cayirci, "Wireless Sensor Networks", A Survey. Computer Networks, vol. 38, no. 4, (2002), pp. 393-422.

[7] S. Irmak and D. Haman, "Performance of Watermark Granular Matrix Sensor in Sandy Soils", Applied Engineering in Agriculture, vol. 6, no. 17, (2001), pp. 787-795.

[8] F. I. Lamiaa and A. F. S. Hesham, "Applying Clustering Techniques in Hybrid Network in the Presence of 2D and 3D Obstacles", Computing and informatics, vol. 32, no. 6, (2013), pp. 1170-1191.

[9] J. R. Coen, K. Henk and K. Leon, "A new wireless underground network system for continuous monitoring of soil water contents", Water resources research, vol. 45, no. 36, (2009), pp. 36-44.

[10] O. Green, E. S. Nadimi and V. Blanes, "Monitoring and modeling temperature variations inside silage stack using novel wireless sensor networks", Computers and Electronics in Agriculture, vol. 69, no. 1, (2009), pp. 149-157.

[11] J. A. Lopez, F. Soto and J. Suardiaz, "Wireless sensor networks for precision horticulture in Southern Spain", Computers and Electronics in Agriculture, vol. 68, no. 3, (2009), pp. 25-35.

[12] E. Berman, G. Calinescu, C. Shah and A. Zelikovsky, "Power efficient Monitoring Management in Sensor Networks", Proceedings of IEEE Wireless Communication and Networking Conference, (2004).

[13] M. K. Watfa, H. Al-Hassanieh and S. Salmen, "A novel solution to the energy hole problem in sensor networks", Journal of network and computer applications, vol. 36, no. 2, (2013), pp. 949-958.

[14] J. Carle and D. SimPlot-Ryl, "Energy Efficient Area Monitoring by Sensor Networks", IEEE Computer Magazine, vol. 37, no. 2, (2004), pp. 40-46.

[15] T. Rafael, M. O. Juan, P. Maurizio and D. Jose, "A Topology-Independent Mapping Technique for Application-Specific Networks-on-Chip", Computing and informatics, vol. 31, no. 5, (2012), pp. 939970.

[16] A. Chehri, P. Fortier and P. M. Tardif, "Application of Ad-hoc sensor networks for localization in underground mines", Wireless and Microwave Technology Conference, (2006). 
[17] L. Li and X. M. Wen, "Energy Efficient Optimization of Clustering Algorithm in Wireless Sensor Network", Journal of electronics \& information technology, vol. 30, no. 4, (2008), pp. 966-969.

[18] H. R. Bogena, J. A. Huismana, H. Meierb, U. Rosenbauma and A. Weuthena, "Hybrid wireless underground sensor networks: Quantification of signal attenuation in soil", Vadose Zone Journal, vol. 8, no. 3, (2009), pp. 755-761.

[19] E. Shih, S. Cho, N. Ickes, R. Min, A. Sinha, A. Wang and A. Chandrakasan, "Physical Layer Driven Protocol and Algorithm Design for Energy-Efficient Wireless Sensor Networks", Proceedings of ACM MOBICOM, (2001), pp. 272-286.

[20] D. Xin and C. Mehmet, "Spatio-temporal Soil Moisture Measurement with Wireless Underground Sensor Networks", Ad hoc Networking Workshop, (2010).

[21] A. Sheth, K. Tejaswi and P. Mehta, "Senslide: A Sensor Network Based Landslide Prediction System", The 3rd International Conference on Embedded Networked Sensor Systems, (2005), pp. 280-281.

[22] G. W. Allen, K. Lorincz, M. Welsh and O. Marcillo, "Deploying a Wireless Sensor Network on An Active Volcano", IEEE Internet Computing, vol. 10, no. 2, (2006), pp. 18-25.

[23] A. F. Catherine, T. Charles and B. Gabriele, "A new horizon protocols for topsoil characterization in Canada", 19th World Congress of Soil Science, vol. 6, (2010), pp. 9-12.

[24] R. Aqeel, A. Abu and I. Noman, "A review of wireless sensors and networks applications in agriculture", Computer Standards \& Interfaces, vol. 1, no. 4, (2011), pp. 259-363.

[25] Y. Kima, Y. M. Yang and W. S. Kang, "On the Design of Beacon based Wireless Sensor Network for Agricultural Emergency Monitoring Systems", Computer Standards \& Interfaces, vol. 5, no. 4, (2011), pp. 32-39.

[26] M. J. Tiusanen, "Attenuation of a Soil Scout radio signal", Biosystems Engineering, vol. 90, no. 2, (2005), pp. 127-133.

[27] G. Stuber, "Principles of Mobile Communication", Klumer Academic Publishers, (2001).

[28] S. Ramo, J. Whinnery and D. T. Van, "Fields and Water for Communications Electronics", John Wiley and Son, New York, (1994).

[29] J. Wait, "Electromagnetic Wave Theory", Harper and Row, New York, (1985).

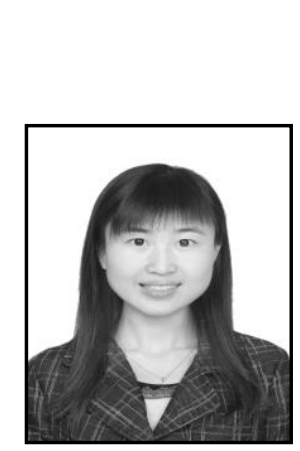

\section{Authors}

Xiao Q. Yu received the B.S. degree from Department of Information Engineering, Lanzhou University of Finance and Economics, Lanzhou, China in 2006. She received her M.S. degree and Ph.D. degree from Department of Mechanical and Electric Engineering and Department of Water Resources and Architectural Engineering, Northwest A \& F University, Shaanxi, China in 2009 and 2013, respectively.

Currently, she is pursuing post doctor degree from Department of Water Resources and Architectural Engineering under the supervision of Prof. Wen T. Han. Her current research interests are in Agricultural Water-Soil Engineering and Wireless Sensor Networks.

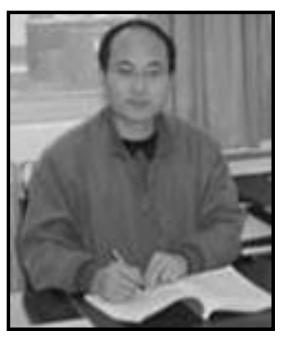

Wen T. Han received his B.S. degree from Department of Mechanical and Electric Engineering, Northwest Agriculture University, Shaanxi, China in 1996. M.S. and Ph.D. degree from Department of Mechanical and Electric Engineering, Northwest A \& F University, Shaanxi, China in 1999 and 2004, respectively.

\section{Working Experiences:}

2005-present: researcher, Institute of Soil and Water Conservation of Chinese Academy of Sciences Northwest A \& F University, National Engineering Research Center for Water Saving Irrigation at Yangling.

2004-2005: Assistant Professor, Department of Mechanical and Electric Engineering, Northwest A \& F University. 
2001-2004: A lecturer, Department of Mechanical and Electric Engineering, Northwest A \& F University.

\section{Research Interests:}

Information monitoring of crop and environment; Intelligent control for precise irrigation; water distribution Simulation of sprinkler irrigation; Development of nozzle

Currently, he published academic papers more than 30, including SCI and EI articles 16; the national invention patent 6.

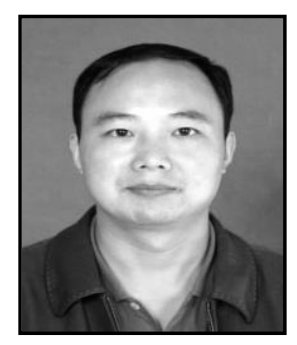

Zeng L. Zhang received his B.S. degree from Department of Mechanical and Electric Engineering, Harbin institute of Technology, Harbin, and M.S. degree from Department of Mechanical and Electric Engineering, Northwest A \& F University, Shaanxi, China in 2000 and 2007, respectively.

Currently, he is a teacher in Department of Mechanical and Electric Engineering, Northwest A \& F University, Shaanxi. He is pursuing Ph.D. degree under the supervision of Prof. Pu T. Wu. His current research interests are in Agricultural Water-Soil Engineering and Wireless Sensor Networks. 
International Journal of $u-$ and $e-$ Service, Science and Technology Vol.8, No. 7 (2015) 